very far the author succeeds in convincing the reader of the advantage resulting from attacking a problem in mechanics by appropriate mathematical tools.

Matrix theory occupies about one third of the book. A brief account of matrix algebra (including the Cayley-Hamilton theorem) is followed by a discussion of power series in matrices and of the calculus of matrix-valued functions. Applications are given to the theory of small oscillations around a point of stable equilibrium, to the theory of aircraft flutter and to elastic deformation theory.

Tensor calculus is developed primarily for the Euclidean threespace. The discussion centers around curvilinear coordinates, the metric tensor and covariant differentiation. As a first application the fundamental equations of mathematical physics are written in general curvilinear coordinates. A brief chapter on fluid dynamics is followed by a comparatively extensive treatment of tensor methods in the theory of elasticity. A distinctive feature of these chapters is the author's determination not to limit himself to infinitesimal deformations. The last two chapters are devoted to tensor calculus in Riemannian spaces with applications to classical mechanics and boundary layer theory.

The volume is based on a series of lectures given to a group of research engineers. It could be well used in a graduate or senior undergraduate course for engineering students. The exercises are not numerous but are selected skillfully. A few awkward expressions and slips of the pen (for instance on p. 32, 1.27, p. 34, 1.11, p. 41, 1.10, p. $88,1.20)$ do not detract materially from the value of this enthusiastically written and useful book.

\title{
LipMAN Bers
}

Theory and application of Mathieu functions. By N. W. McLachlan.

New York, Oxford University Press, 1947. 9+401 pp. $\$ 12.50$.

Part I of this book contains a comprehensive treatment of analytical and numerical methods which have been successfully used to obtain solutions of the various forms of Mathieu's differential equation, satisfying various conditions, and for complex as well as real values of the independent variable. It discusses also the theoretical background underlying these methods. A large part, estimated by the author as one third, of this material is new, filling gaps by extending over the whole field methods which had proved useful in part of it; the book will therefore be very useful to a reader who wants to make a new application without having to extend the theory.

The subjects covered include the integral equations and relations satisfied by the solutions; the distribution of their zeros; the periodic 
properties of the solutions and their expansions in Fourier series and the corresponding expansions of solutions that are not periodic; and the expansions in series of Bessel functions and of products of Bessel functions and the asymptotic expansions for large argument values. Powerful methods are given for computing the characteristic numbers and the coefficients in the expansions.

The applications fall naturally into two classes. Parametric resonance in dynamical systems where Mathieu's equation is to be considered the simplest special form of Hill's equation-also treated in Part I; and the solution of two-dimensional problems by separation of variables in elliptic coordinates, described for the wave equation also in Part I. The representative applications in Part II of the first class include treatments of amplitude distortion, frequency modulation, and sub-harmonics; of the second class the vibrations of elliptical membranes, plates, and lakes; eddy currents and thermal diffusion in elliptic cylinders; the propagation of electromagnetic waves in elliptical wave guides; and the diffraction of waves by elliptical cylinders. These, and the other applications given, should enable the reader to apply the theory to new cases as they arise.

The only large part of the theory not given is that connected with the treatment of the equation in terms of the singular points of its algebraic form.

The book is beautifully printed and a pleasure to read; it fulfills a long felt want and will take its place alongside Watson's Bessel functions as a necessary part of the equipment of an applied mathematician.

L. H. ThомаS

Algèbre et analyse linéaires. By A. Lichnerowicz. Paris, Masson, 1947. 316 pp. $800 \mathrm{fr}$.

This book is an introduction to linear mathematics for physicists based on lectures given by the author at Strasbourg. It is designed to fill in part the gap left in French scientific literature by the absence of works in French similar to the German treatises of Courant and Hilbert and of Frank and Mises.

The book has two parts, one on linear algebra and one on linear analysis, and each part is divided into four chapters. The first three chapters of Part I and the last three chapters of Part II present the standard elementary material on linear operators and bases in vector spaces with applications to the theory of expansions in orthogonal systems of functions and to the theory of integral equations. The middle two chapters present the algebra and analysis leading up to 